\title{
GW23-e2716 DIABETES MELLITUS, THE IMPORTANT CARDIOVASCULAR RISK FACTOR OF PREMATURE MYOCARDIAL INFARCTION IN WOMEN
}

doi:10.1136/heartjnl-2012-302920a.287

${ }^{1}$ Eliverta Zera, ${ }^{2}$ Elizana Zaimi, ${ }^{1}$ Mimoza Metalla, ${ }^{1}$ Stela Prifti, ${ }^{3}$ Eliverta Zera. ${ }^{1}$ Cardiology Regional Hospital Durres; ' 2 Department of Epidemiology 'Mother Teresa' University Tirane, ${ }^{3}$ Regional Hospital Durres

Objectives Acute Myocardial infarction (AMI) is the leading cause of mortality and morbidity both in men and women. The frequency and profile of conventional risk factors differs in women compared to men and in different age groups.

The Aim To evaluate the conventional cardiovascular risk factors in young women with AMI and to compare it with other age and sex groups, in Durres population, Albania.

Methods This study evaluated the prevalence of classic CHD risk factors in female patients with AMI aged $\leq 56$ and compared it with female patients with AMI more than 56 years old and also with the male patients with AMI. 286 patients with AMI who presented in the Cardiology Department, Regional Hospital of Durres, Albania between January 2011 to March 2012 were included in the study. ( 9 pts female age $\leq 56,67$ pts female age $\geq 56$ and 210 pts male). All subjects underwent detailed evaluation of cardiovascular risk factors. Patients were screened for the presence of diabetes mellitus, hypertension smoking and history of ischaemic heart disease in their first degree relatives. Lipid profile was recorded from the investigation chart of every patient.

Results Diabetes was detected to be the most frequent coronary risk factor $(29.2 \%)$ in young women with AMI. The prevalence of diabetes was $23.4 \%$ in women with AMI. Women with premature AMI ( $\leq 56$ years old) were found to have a higher prevalence of diabetes compared to older women $(29.2 \%$ vs $18.1 \%)$ p $<0.01)$. The number of diabetics was significantly higher in women than men (23.4\% vs $13.9 \%)$. The number of diabetics was significantly higher in younger women than younger men (age $\leq 56,29.2 \%$ vs $13.3 \%$ ). Hypertension was second risk factor in younger female group and the most common risk factor in older female group $(20.8 \%$ vs $33.3 \%$ ). Cigarette smoking was found to be the least common risk factor in the younger female group but the most common in the younger male $(27.5 \%$ vs $64.1 \%$ ). Mean age in men was 60.29 \pm 10.84 , mean age in women was $68.88 \pm 8.51(p<0.001)$. The mean age of female AMI patients was only 8.6 years more than male MI patients, which is less than the 10 years delay of MI in females reported in the literature. There were not statistical differences between women and men for history of ischaemic heart disease and lipid profile.

Conclusions Our study show a higher prevalence of diabetes in young females compared with both older females and males with AMI. The higher prevalence of DM in young females may be associated with the decreasing difference of mean age between female 
and male patients with MI. This data may be useful in directing primary and secondary preventive measures. 